\title{
New Developments in Geotechnical Earthquake Engineering
}

\author{
Yang Changwei, ${ }^{1}$ Su Tianbao, ${ }^{2}$ Zhang Jianjing, ${ }^{1}$ and Du Lin ${ }^{1}$ \\ ${ }^{1}$ School of Civil Engineering, Key Laboratory of Transportation Tunnel Engineering, Ministry of Education, \\ Southwest Jiaotong University, Chengdu 610031, China \\ ${ }^{2}$ Henan University of Urban Construction, Pingdingshan, Henan 467036, China \\ Correspondence should be addressed to Zhang Jianjing; yangchangwei56@163.com
}

Received 21 February 2014; Revised 31 March 2014; Accepted 17 April 2014; Published 7 July 2014

Academic Editor: George Z. Kyzas

Copyright (c) 2014 Yang Changwei et al. This is an open access article distributed under the Creative Commons Attribution License, which permits unrestricted use, distribution, and reproduction in any medium, provided the original work is properly cited.

Based on the review on the advances of several important problems in geotechnical seismic engineering, the authors propose the initial analysis theory of time-frequency-amplitude (known as TFA for short), in an effort to realize the organic combination of time and frequency information and develop a groundbreaking concept to the traditional idea in the geotechnical seismic engineering area.

\section{Review on the Present Main Defect in the Geotechnical Earthquake Engineering}

Geotechnical seismic engineering is an important area in geotechnical engineering, and its topic is to resolve the seismic problems related to geotechnical engineering. With development for more than 50 years, the time domain analysis theory and the frequency analysis theory have been generally established in the geotechnical seismic engineering [1-6]. However, a common problem still exists in the time domain analysis theory and the frequency domain theory, which is that the research methods for geotechnical seismic engineering are still the separate application of time domain or frequency domain, and no combination of above two is considered. [7-11]. For example, time domain analysis theory can only consider the time histories of acceleration, velocity, and displacement but not the frequency contents of ground motions. Frequency domain analysis theory can only consider the frequency contents of ground motions, while time histories of acceleration, velocity, and displacement are not included. Seismic wave is however a very complex nonstationary signal, whose amplitudes and frequencies change with time. Present outcomes in the time domain theory and the frequency domain theory cannot reflect synthetically the characteristics of geotechnical seismic engineering, very close to an old Chinese saying "The blind man feels an elephant-to take a part for the whole." Therefore, research on the timefrequency-amplitude analysis theory considering the time, frequency, and amplitude can be an important task, which will be a new direction of geotechnical seismic engineering.

\section{Brief Introduction of the Signal Analysis Technologies}

Fourier transform and wavelet transform are two seismic signal analysis technologies [12]. Fourier transform is a steady-state analysis technology and suitable for frequency domain analysis. Wavelet transform has some timefrequency domain resolution ability, but it is hard to carry out an accurate time-frequency analysis [13] because of uncertainty principle. Based on the Hilbert transform, Huang et al. proposed a new signal analysis method for nonstationary signal [14] called Hilbert-Huang transform (HHT). The approach can perform linearized and stabilized analysis for nonlinear and nonstationary signals, initial data in the analysis can be retained, and energy leaking can be avoided. Studies in the past showed that the results from HHT can reflect true cases. The concrete calculation principle of Hilbert-Huang transform is shown as follows: generally, a nonstationary signal can be decomposed into a series of 


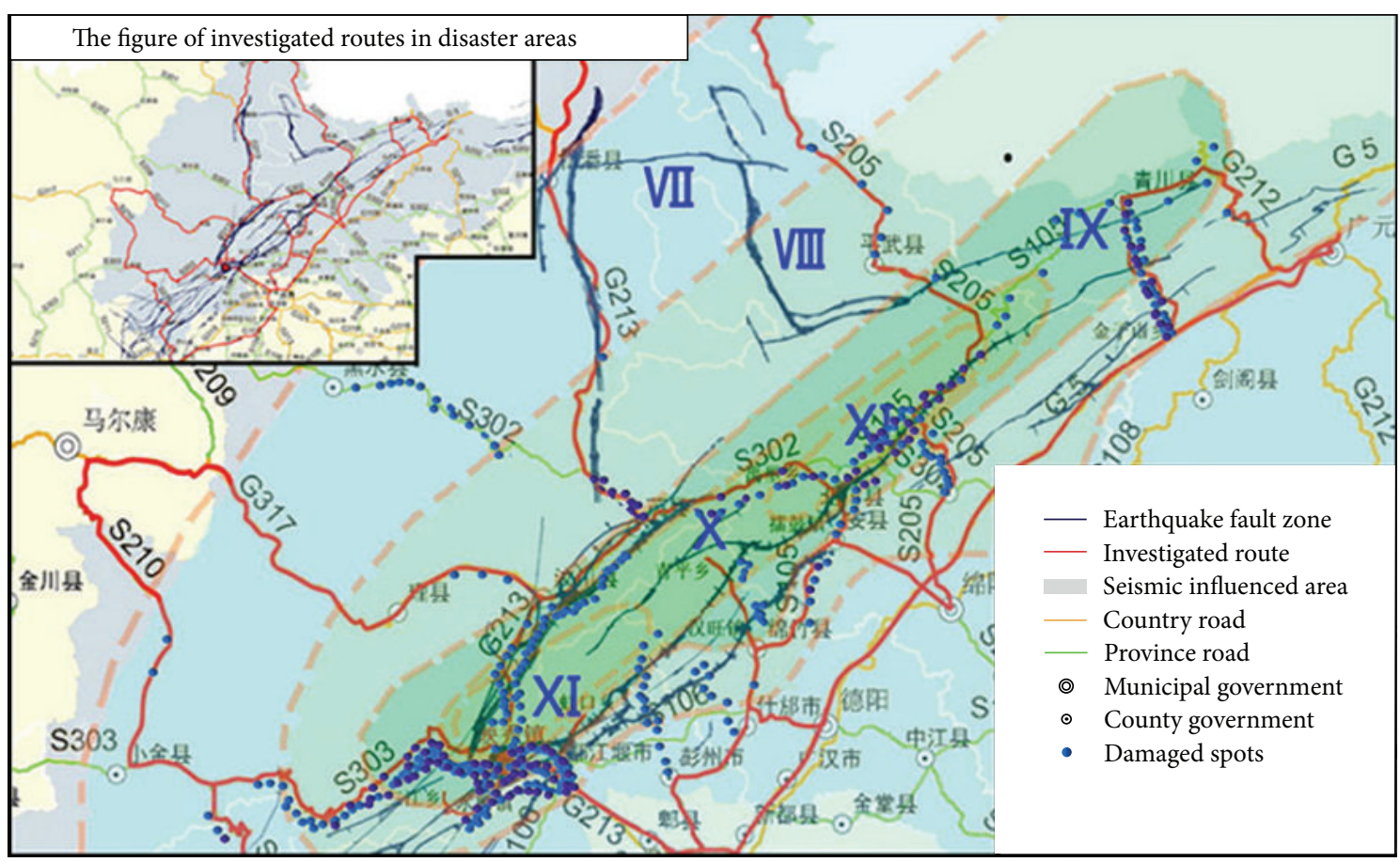

FIGURE 1: Roads investigated and seismic intensity.

intrinsic mode function (known as IMF for short) components by using ensemble empirical mode decomposition (known as EEMD for short) method. The instantaneous frequency of each IMF will be derived by the Hilbert-Huang transform, and then the Hilbert spectrum will be obtained by integrating all the instantaneous frequency spectrums. The analytical signal, amplitude function, phase function, and instantaneous frequency function can be obtained by using the Hilbert-Huang transform. The computational formulas are shown in

$$
\begin{gathered}
Z(t)=c(t)+j H[c(t)]=a(t) e^{j \phi(t)}, \\
a(t)=\sqrt{c^{2}(t)+H^{2}[c(t)]}, \\
\phi(t)=\arctan \frac{H[c(t)]}{c(t)}, \\
f(t)=\frac{1}{2 \pi} \frac{d \phi(t)}{d(t)} .
\end{gathered}
$$

From the above formulas, it is known that both amplitude and frequency are the function of time. If the amplitude is calculated by using a combined time and frequency domain approach, the Hilbert spectrum can be obtained, as shown in (2). The marginal spectrum and the instantaneous frequency spectrum can be obtained by using (3) and (4). The Hilbert spectrum can be obtained by integrating amplitude squared, as shown in (5).

Consider

$$
H(\omega, t)=\operatorname{Re} \sum_{i=1}^{n} a_{i}(t) e^{j \phi_{i}(t)},
$$

$$
\begin{aligned}
h(\omega) & =\int_{0}^{T} H(\omega, t) d t, \\
I E(t) & =\int_{\omega} H(\omega, t) d t, \\
E S(\omega) & =\int_{0}^{T} H^{2}(\omega, t) d t .
\end{aligned}
$$

\section{New Development in the Geotechnical Earthquake Engineering}

Up to now, our team has conducted a field investigation along the road and railway nearly $3000 \mathrm{~km}$ in 5.12 Wenchuan earthquake-stricken areas (see Figure 1), a number of field monitoring of typical slopes, more than twenty shaking table tests, a number of numerical simulations and theoretical studies $[15,16]$; some shaking tabletest models are shown in Figures $2,3,4,5$, and 6 .

On the basis of extensive studies, several important problems in the geotechnical seismic engineering are studied, including analysis methods of seismic responses of the site, analysis methods of seismic responses and seismic stability of the retaining structures, analysis methods of seismic responses of the slope, analysis methods of the landslide mechanism, and the analysis method of seismic stability of the slope. With the help of above-mentioned signal analysis skills in the aerospace area and signal analysis area (e.g., Hilbert-Huang transform), our team has conducted a series of analyses on civil structure depending on the proposed time-frequency-amplitude (known as TFA for short) analysis theories, which are comprised of horizontal layers, inclined 


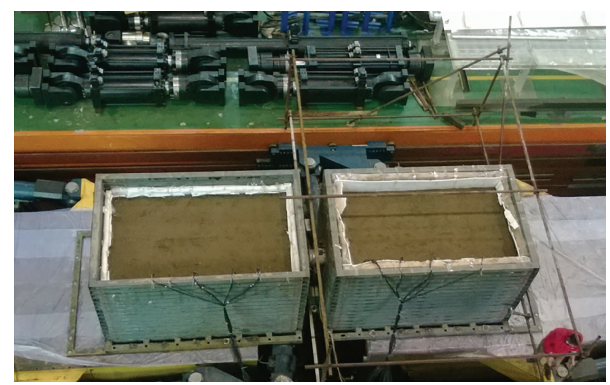

FIGURE 2: Shaking table test of site under nonuniform seismic excitation.

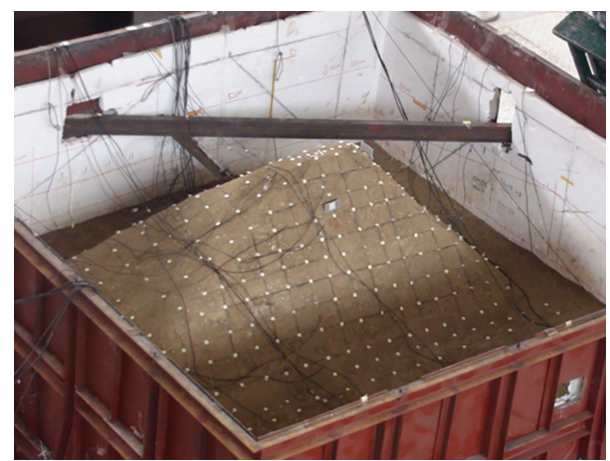

FIGURE 3: Shaking table test of rock slope.

layers, gravity retaining wall, reinforced retaining wall, slopes, and landslide mechanism $[17,18]$. Our team gives the initial prototype of TFA analysis theories in geotechnical seismic engineering, which realizes the organic combination of time and frequency information, and develops a groundbreaking concept to the traditional idea in the geotechnical seismic engineering area.

\section{Essence of the Time-Frequency-Amplitude Analysis Theories}

The time-frequency-amplitude analysis theories give reasonable considerations to the time-frequency characteristics of seismic waves, mainly reflected in the elastic displacement magnitude and the frequency of the wave vector. This time, this paper selects Wolong earthquake wave (as shown in Figure 7) to make an explanation; procedures are as follows: first, Wo-Long wave is decomposed into intrinsic mode functions (IMF) using the empirical mode decomposition (EMD) method. The acceleration time histories of each IMF can be seen in Figure 8 and the instant frequency time histories of each IMF can be seen in Figure 9. Based on the acceleration time histories and instant frequency time histories of each IMF, bring them into the related formulas in order to get the stress time histories of each IMF; finally, sum the stress time histories together in order to obtain the total results.

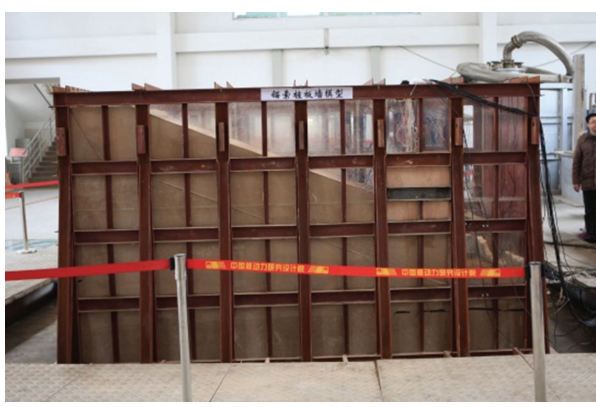

FIGURE 4: Shaking table test of retaining wall.

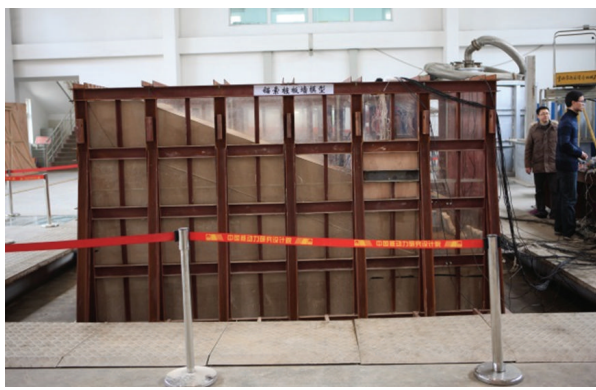

FIGURE 5: Shaking table test of antisliding pile.

\section{Advantages of the Time-Frequency Analysis Method of Seismic Safety of the Rock Slope}

Comparisons with pseudostatic methods using the various countries' earthquake resistant design codes, TFA analysis theory of seismic stability of rock slope is capable of considering the time, frequency, and amplitude of seismic wave, and then a brief introduction of the above-mentioned theory is made as follows.

In the TFA analysis theory of seismic stability of rock slope, the calculated model is shown in Figures 10 and 11. In the model, the sliding mass was divided into seven slices, and an angle of $\theta$ between the tangent and horizontal directions at any point $\mathrm{A}$ was obtained at the middle of a slice. Figure 11 shows the reflection and refraction model at point $\mathrm{A}$. The mechanical parameters in the upper medium (bedrock) are $\rho_{1}, C_{p 1}, C_{s 1}$, and $G_{1}$ (density, $P$ wave speed, $S$ wave speed, and shear modulus) and $\rho_{2}, C_{p 2}, C_{s 2}$, and $G_{2}$ in the lower medium (colluvial soils), and the I-I stands for the sliding surface. When transverse wave $S^{1}$ reaches the I-I surface, some new waves are produced, for example, reflected transverse wave $S^{2}$, reflected longitudinal wave $S^{3}$, refracted transverse wave $S^{4}$, and refracted longitudinal wave $S^{5} . \alpha_{1}, \alpha_{1}^{\prime}, \beta_{1}$, and $\beta_{1}^{\prime}$ stand for the incident angle of the incident transverse wave, the reflected angle of the reflected longitudinal wave, the refracted angle of the refracted transverse wave, and the refracted angle of the refracted longitudinal wave. Note that the reflected angle of the reflected transverse wave is the same as the incident angle of the incident transverse wave.

After the derivation, the stress state at any point can be calculated according to (6) and (7). And then, the stress state at whole sliding surface can be calculated according to (8). At 


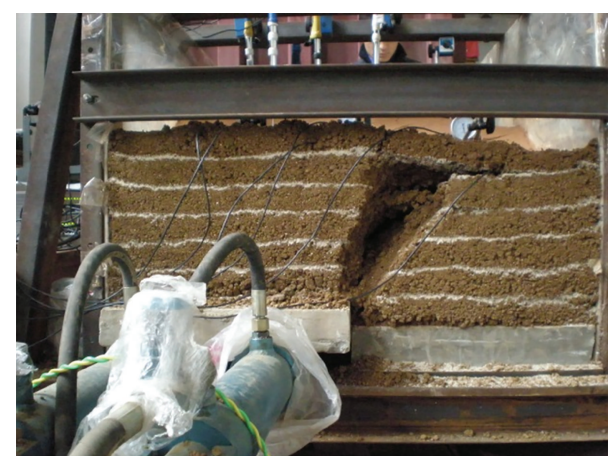

FIGURE 6: Simulation test of fault slip.

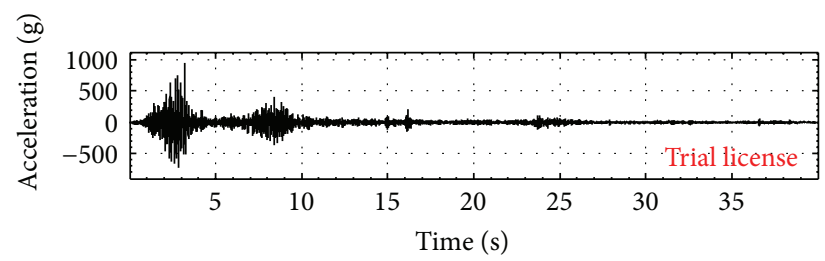

FIgURE 7: Time history of the Wenchuan-Wolong seismic wave.

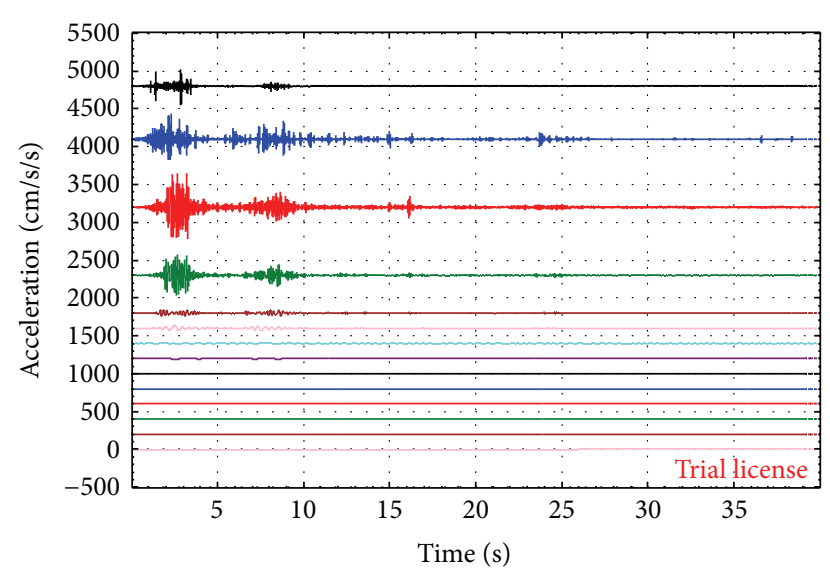

FIGURE 8: Acceleration time history of IMF.

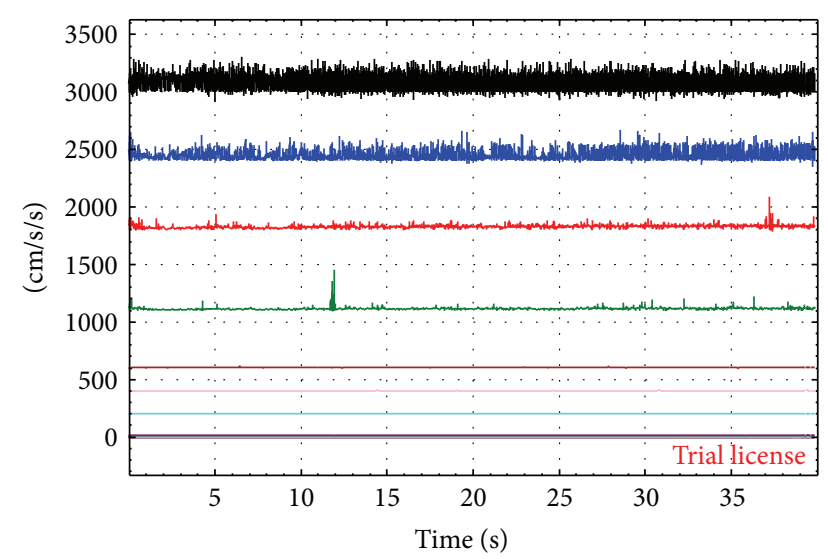

FIGURE 9: Instant frequency time history of IMF.

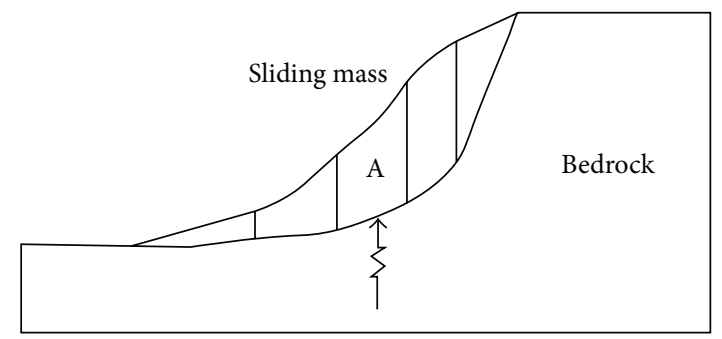

FIGURE 10: Generalized model of the bedrock-regolith slope.

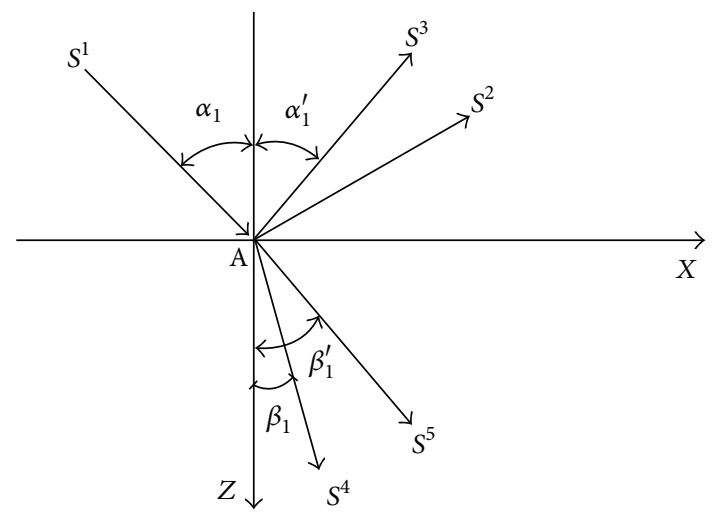

FIGURE 11: Analysis model of reflection and refraction at any point A.

last, the seismic stability of slope can be estimated according to (9).

Consider

$$
\begin{aligned}
\tau_{s}=\tau_{0}+\mu_{1}[ & S_{0}^{1} \cdot k_{z}^{11} \cdot \cos \alpha_{1}-S_{0}^{2} \cdot k_{z}^{21} \cdot \cos \alpha_{1} \\
& +S_{0}^{3} \cdot k_{z}^{31} \cdot \sin \alpha_{1}^{\prime}-S_{0}^{1} \cdot k_{x}^{11} \cdot \sin \alpha_{1}-S_{0}^{2} \cdot k_{x}^{21} \\
& \left.\cdot \sin \alpha_{1}-S_{0}^{3} \cdot k_{x}^{31} \cdot \cos \alpha_{1}^{\prime}\right]
\end{aligned}
$$

$$
\begin{aligned}
& \sigma_{0} \tan \varphi+C \\
&=\left\{\lambda_{1}[\right. S_{0}^{1} \cdot k_{x}^{11} \cdot \cos \alpha_{1}-S_{0}^{2} \cdot k_{x}^{21} \\
&\left.\cdot \cos \alpha_{1}+S_{0}^{3} \cdot k_{x}^{31} \cdot \sin \alpha_{1}^{\prime}\right]+\left(\lambda_{1}+2 \mu_{1}\right) \\
& \times {\left[-S_{0}^{1} \cdot k_{z}^{11} \cdot \sin \alpha_{1}-S_{0}^{2} \cdot k_{z}^{21} \cdot \sin \alpha_{1}\right.} \\
&\left.\left.-S_{0}^{3} \cdot k_{z}^{31} \cdot \cos \alpha_{1}^{\prime}\right]+\sigma_{0}\right\} \tan \varphi+C .
\end{aligned}
$$

In (6) and (7), $S_{0}^{1}$ stand for the displacement of incident transverse wave, $S_{0}^{2}$ stand for the displacement of reflected transverse wave, $S_{0}^{3}$ stand for the displacement of reflected longitudinal wave, $S_{0}^{4}$ stand for the displacement of refracted transverse wave, and $S_{0}^{5}$ stand for the displacement of refracted longitudinal wave; $k_{x}^{i 1}, k_{z}^{i 1}$ stand for the wave vectors in the $X$ and $Z$ directions of the incident, reflected, and the 


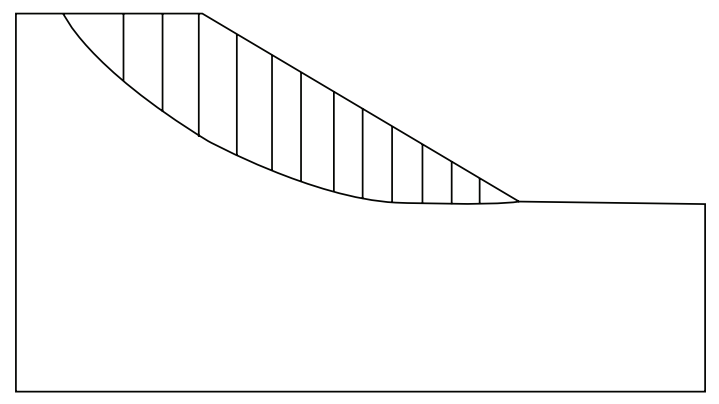

Figure 12: Calculated model.

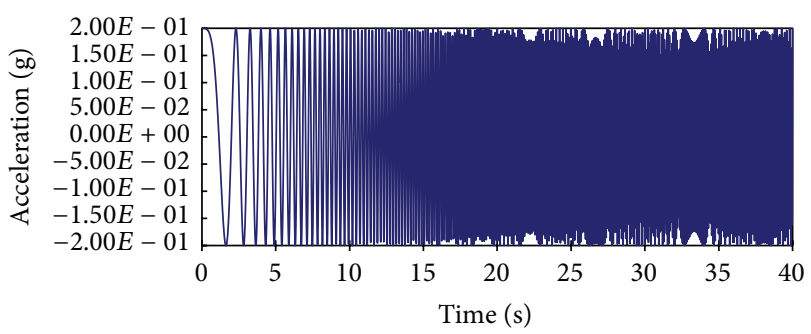

Figure 13: Time history of sine wave.

refracted waves, respectively; $C$ stand for the cohension of the sliding mass and $\varphi$ stand for the internal friction angle.

Consider

$$
\begin{gathered}
F_{s}=\sum_{i=1}^{n} \tau_{s i} \cdot d A_{i} ; \\
F_{r}=\sum_{i=1}^{n}\left(\sigma_{0 i} \cdot \tan \varphi_{i}+C_{i}\right) \cdot d A_{i}, \\
K=\frac{F_{r}}{F_{s}}<K_{0} \quad \text { risk; } \\
K=\frac{F_{r}}{F_{s}}>K_{0} ; \quad \text { safety. }
\end{gathered}
$$

In (8) and (9), $\tau_{s i}$ represents the sliding shear stress at point $i$; $A_{i}=0.5\left(L_{i-1}+L_{i+1}\right) \times 1$ is the slice area, $L_{i-1}$ is the width of the $i-1$ th slice and $L_{i+1}$ is the width of the $i+1$ th slice; $\sigma_{0 i}$ represents the normal stress at point $i ; \varphi_{i}$ represents the frictional angle at point $i$; $C_{i}$ represents the cohesion at point $i$.

According to the above-mentioned method, a simple example is used to illustrate the advantage of the TFA analysis theory: model for calculation is shown in Figure 12. Figures 13 and 14 give the adopted sine wave whose instantaneous frequencies gradually increase with time and the calculated parameters can be seen in Table 1 .

After the calculation is done, results of TFA analysis method are shown in Figure 15, indicating that the slope would fail after $T=10.0 \mathrm{~s}$. The result of pseudostatic method shows that the surplus sliding force is $-47 \mathrm{kN}$ and means that the slope is stable. While the slope is proven to fail in the numerical simulation, which is consistent with the result of

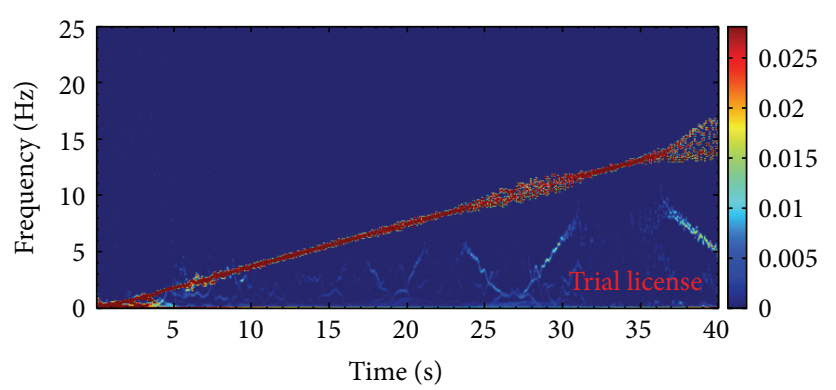

FIGURE 14: Hilbert spectrum of sine wave.

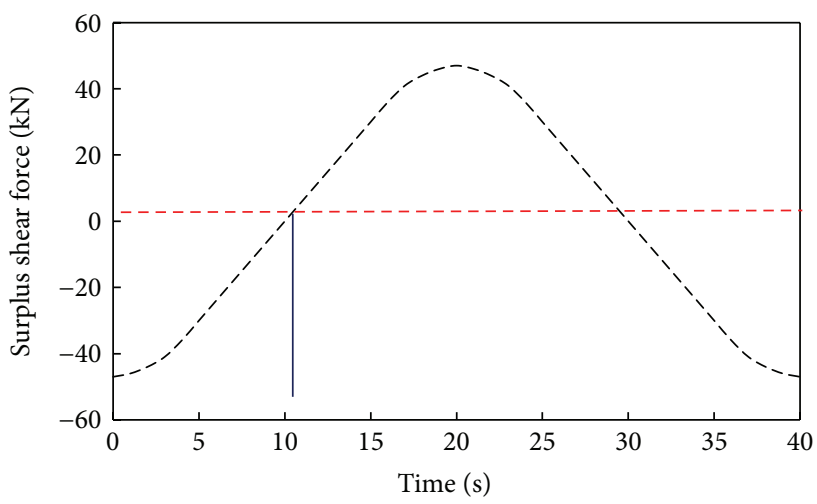

Figure 15: Result of TFA analysis method.

TFA analysis method shown in Figure 16, this phenomenon can be elucidated as follows.

(i) At $T=10.0 \mathrm{~s}$, the instantaneous frequency of the seismic wave is $4.9 \mathrm{~Hz}$ and the natural frequency calculated according to the literature [17] is $6.8 \mathrm{~Hz}$, with both being very close to each other, leading to a further resonance. However, the instantaneous frequency of the input seismic wave reaches $6.8 \mathrm{~Hz}$ at $T=18.2 \mathrm{~s}$, and the resonance shows the strongest value, which leads to the maximum surplus shear force. Then the resonance gradually decreases with difference between the frequencies of the input wave and the natural frequency goes up, and the surplus shear force gradually decreases in response.

(ii) Time-frequency analysis method and numerical method both not only can consider the effect of PGA on the seismic stability of slope, but also consider the effect of frequency on the seismic stability of slope. But pseudo-static method only can consider the effect of PGA on the seismic stability of slope, which leads to the difference between the calculated results.

Synthesizes the above analysis, we can know that, therefore, the TFA analysis method can solve the fundamental flaw existing in the analysis method using the codes. 
TABLE 1: Physical and mechanics parameters of bedrock, soil layer, and structural plane.

\begin{tabular}{lccccc}
\hline Model & Gravity & Shear wave velocity & Longitudinal wave velocity & Lame coefficients & Shear modulus \\
\hline Bedrock & $30 \mathrm{kN} / \mathrm{m}^{3}$ & $826.19 \mathrm{~m} / \mathrm{s}$ & $3240 \mathrm{~m} / \mathrm{s}$ & $881.89 \mathrm{MPa}$ & $2047.24 \mathrm{MPa}$ \\
Slip mass & $19 \mathrm{kN} / \mathrm{m}^{3}$ & $99.21 \mathrm{~m} / \mathrm{s}$ & $145.34 \mathrm{~m} / \mathrm{s}$ & $6.30 \mathrm{MPa}$ & $6.30 \mathrm{MPa}$ \\
Sliding surface & Normal stiffness & Shear stiffness & Internal friction angle & Tensile strength & Cohesion \\
& $4500 \mathrm{MPa}$ & 2300 & $32^{\circ}$ & $23 \mathrm{kPa}$ & $50 \mathrm{kPa}$ \\
\hline
\end{tabular}

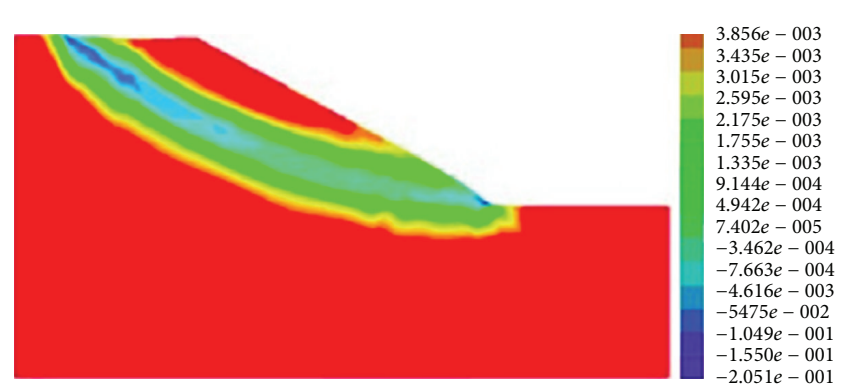

FIGURE 16: Results of numerical simulation.

\section{Application in Practice}

Up to now, the TFA analysis theory has been already used to direct the aseismic designs of geotechnical engineering, containing several kilometers of YaLe expressway, and the contribution to several traffic lines opening.

\section{Conclusions and Future Works}

The main conclusions of this study are as follows.

(i) Based on the above analysis, this paper gives an initial prototype of time-frequency-amplitude analysis theory in the geotechnical seismic engineering. This theory realizes the organic combination of time information and frequency information and gives reasonable considerations to the time-frequency characteristics of seismic waves, which mainly is reflected in the elastic displacement magnitude and the frequency of the wave vector and that is the core content of TFA analysis theories.

(ii) Comparing to the results of TFA analysis theory of rock slope, pseudostatic method, and numerical analysis method shows that the TFA analysis theory can consider the effect of the time and frequency of the input wave on the seismic stability of slope, which solves the fundamental flaw existing in the analysis method using the codes. At the same time, according to the derivation results, we can know that the time-frequency analysis method of rock slope has some universalization and comprehensiveness, which can consider some factors reasonably as follows: (1) initial geostress field; (2) the inner frictional angle, the cohesion, the reflection coefficients, and the refraction coefficients; (3) the physical and mechanical properties of the bedrock and the regolith; and (4) the instantaneous frequency, the incidence angle, the time histories of the acceleration, the velocity, the displacement, and so forth.

(iii) The time-frequency-amplitude analysis theory is built in compliance with the elastic wave theory and geotechnical seismic engineering in which the elastic state of the system is considered. The next works are making the further studies based on the present outcomes; time-frequency-amplitude analysis theory can be thus proposed to consider the nonlinear of the geotechnical engineering and earthquake wave.

\section{Conflict of Interests}

The authors declare that there is no conflict of interests regarding the publication of this paper.

\section{Acknowledgments}

This study is supported in part by the National Science Foundation of China (Contract no. 41030742), NBRP of China (973 Program, 2011CB013605), and Construction and Science and Technology Project of the Ministry of Communications (Contract no. 2013318800020).

\section{References}

[1] D. K. Keefer, "Landslides caused by earthquakes," Geological Society of America Bulletin, vol. 95, pp. 406-421, 1984.

[2] J. N. Hutchinson, "Mechanism producing large displacements in landslides on preexisting shears," Memoir of the Society of Geology of China, vol. 9, pp. 175-200, 1987.

[3] K. Sassa, "Prediction of earthquake induced landslides," in Proceedings of the 7th International Symposium on Landslides, K. Senneset, Ed., vol. 1, pp. 115-132, Balkema, Rotterdam, The Netherlands, 1996.

[4] A. Prestininzi and R. Romeo, "Earthquake-induced ground failures in Italy," Engineering Geology, vol. 58, no. 3-4, pp. 387397, 2000.

[5] M. Lin and K. Wang, "Seismic slope behavior in a large-scale shaking table model test," Engineering Geology, vol. 86, no. 2-3, pp. 118-133, 2006.

[6] M. Chigira, X. Wu, T. Inokuchi, and G. Wang, "Landslides induced by the 2008 Wenchuan earthquake, Sichuan, China," Geomorphology, vol. 118, no. 3-4, pp. 225-238, 2010.

[7] J. Wasowski and V. Del Gaudio, "Evaluating seismically induced mass movement hazard in Caramaico Terme (Italy)," Engineering Geology, vol. 58, no. 3-4, pp. 291-311, 2000.

[8] H. B. Havenith, D. Jongmans, E. Faccioli, K. Abdrakhmatov, and P. Bard, "Site effect analysis around the seismically induced 
Ananevo rockslide, Kyrgyzstan," Bulletin of the Seismological Society of America, vol. 92, no. 8, pp. 3190-3209, 2002.

[9] H.-B. Havenith, A. Strom, D. Jongmans, K. Abdrakhmatov, D. Delvaux, and P. Tréfois, "Seismic triggering of landslides, part A: field evidence from the Northern Tien Shan," Natural Hazards and Earth System Science, vol. 3, no. 1-2, pp. 135-149, 2003.

[10] S. A. Sepúlveda, W. Murphy, R. W. Jibson, and D. N. Petley, "Seismically induced rock slope failures resulting from topographic amplification of strong ground motions: the case of Pacoima Canyon, California," Engineering Geology, vol. 80, no. 3-4, pp. 336-348, 2005.

[11] A. L. Che and X. R. Ge, "Earthquake-induced toppling failure mechanism and its evaluation method of slope in discontinuous rock mass," International Journal of Applied Mechanics, vol. 4, no. 3, Article ID 1250036, 15 pages, 2012.

[12] Z. Zuwu and Y. Lingkan, "Seismic waves scattering in rock interface and energy dissipation characteristics-taking M8.0 Wenchuan Earthquake as an example," Journal of Catastrophology, no. 1, pp. 5-9, 2011.

[13] S. Xu, W. Zheng, Y. Liu, D. Xi, and G. Li, "A preliminary analysis of scale effect of elastic wave propagation in rock mass," Chinese Journal of Geotechnical Engineering, vol. 33, no. 9, pp. 1348-1356, 2011.

[14] N. E. Huang, Z. Shen, S. R. Long et al., "The empirical mode decomposition and the Hilbert spectrum for nonlinear and non-stationary time series analysis," The Royal Society of London A, vol. 454, pp. 903-995, 1998.

[15] J. Zhang, H. Qu, Y. Liao, and Y. Ma, "Seismic damage of earth structures of road engineering in the 2008 Wenchuan earthquake," Environmental Earth Sciences, vol. 65, no. 4, pp. 987-993, 2012.

[16] Y. Changwei and Z. Jianjing, "A prediction model for horizontal run-out distance of landslides triggered by Wenchuan Earthquake," Earthquake Engineering and Engineering Vibration, vol. 12, no. 2, pp. 201-208, 2013.

[17] C. Yang, J. Zhang, and D. Zhou, "Research on time-frequency analysis method for seismic stability of rock slope subjected to SV wave," Chinese Journal of Rock Mechanics and Engineering, vol. 32, no. 3, pp. 483-491, 2013.

[18] C. Yang and J. Zhang, "Landslide responses of high steep hill with two-side slopes under ground shaking," Journal of Southwest Jiaotong University, vol. 48, no. 3, pp. 415-422, 2013. 

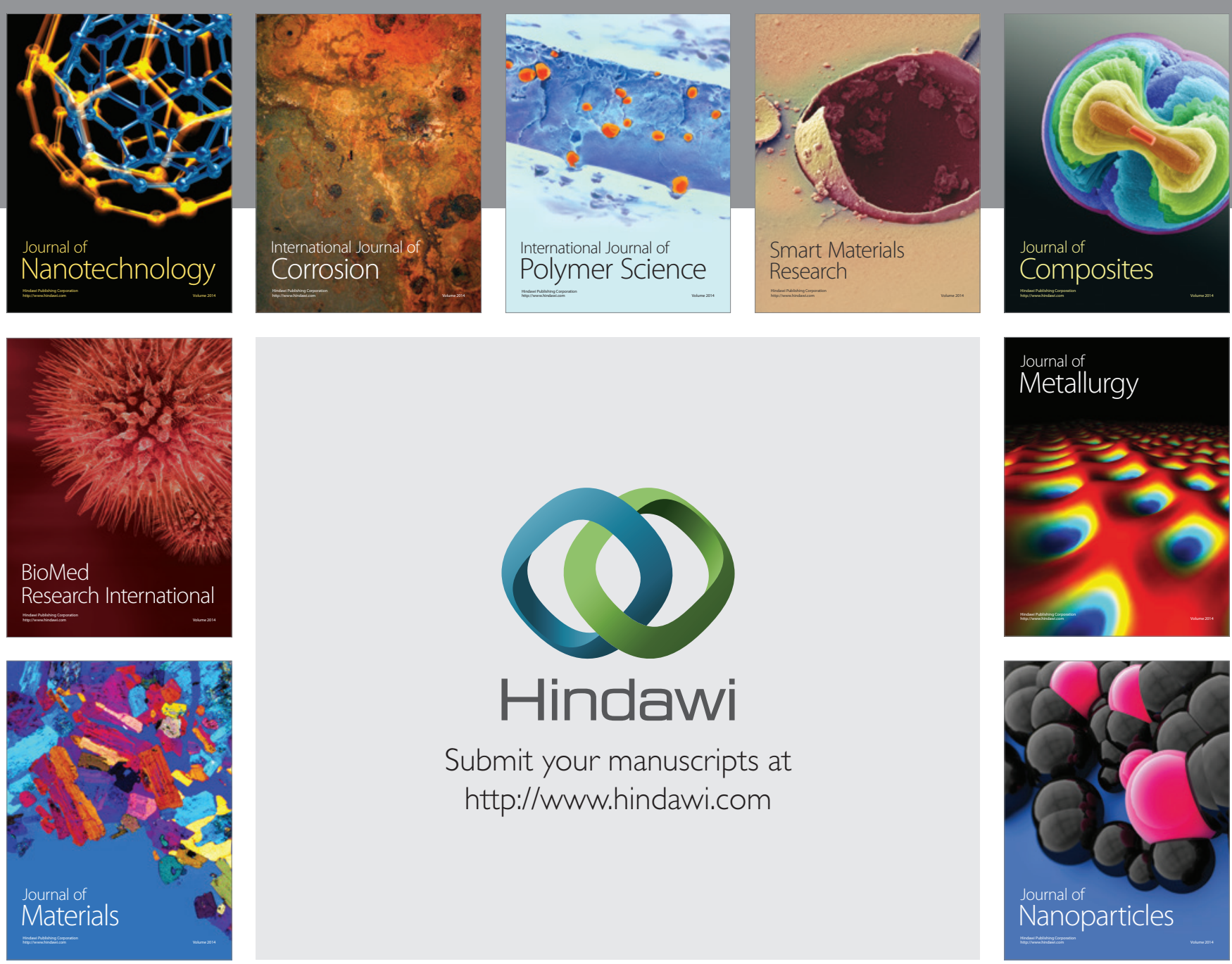

Submit your manuscripts at http://www.hindawi.com
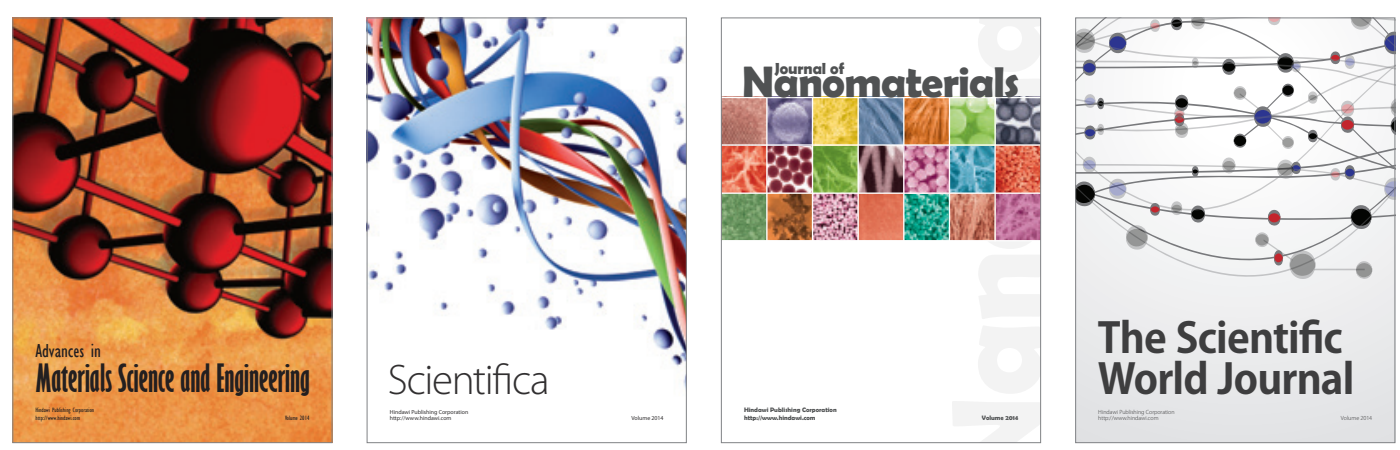

\section{The Scientific World Journal}
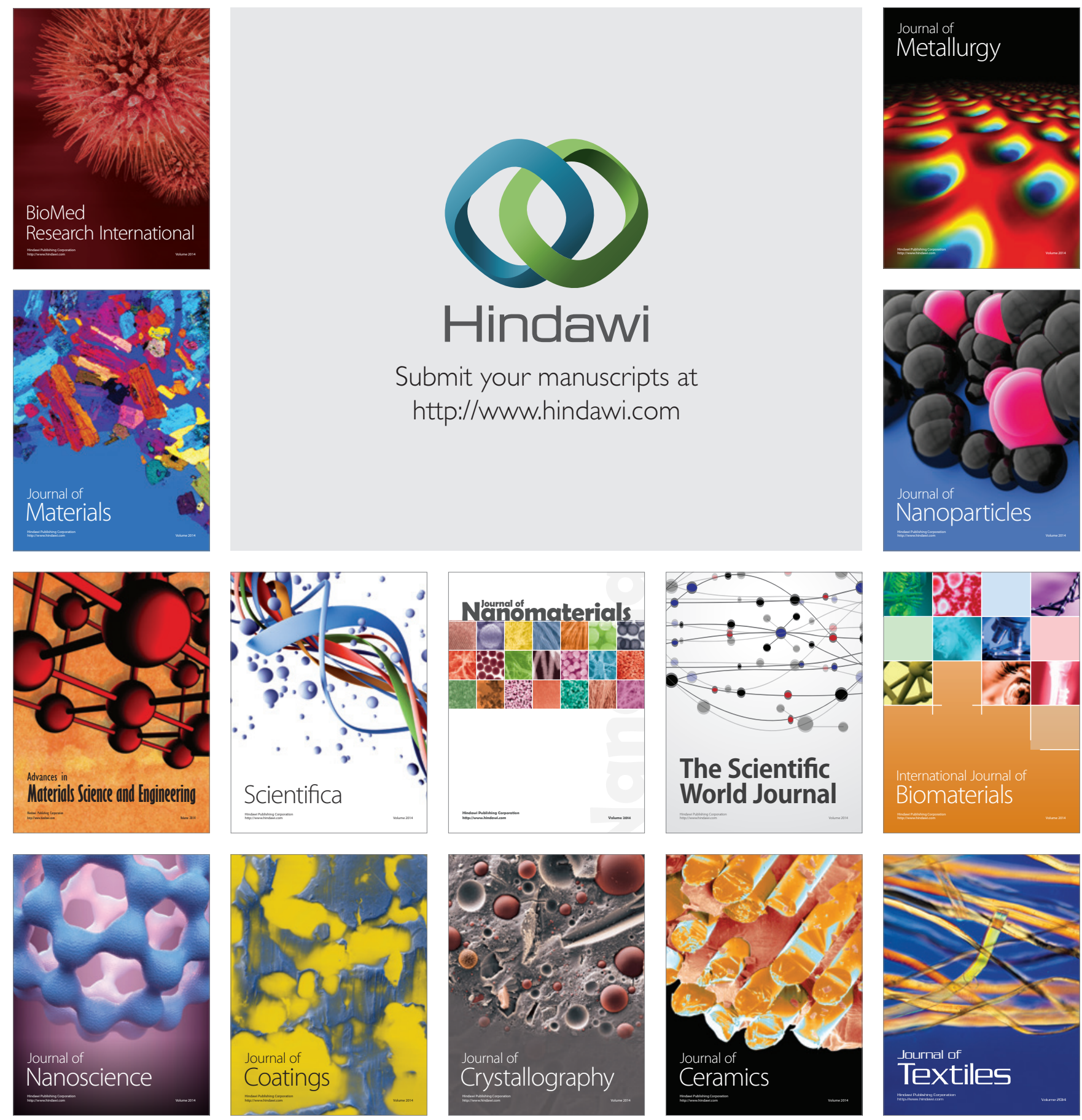